\title{
Treatment of Atrial Fibrillation Using Ablation Index- Guided Contact Force Ablation: A Matching-Adjusted Indirect Comparison to Cryoballoon Ablation
}

\author{
Ahmed Hussein - Dhiraj Gupta - Tom De Potter · Paul Spin • \\ Kiefer Eaton · Laura Goldstein • Maria Velleca • Graça Costa • \\ Daniel Grima · Leena Patel · Giuseppe Stabile
}

Received: November 4, 2019 / Published online: December 21, 2019

(c) The Author(s) 2019

\section{ABSTRACT}

Introduction: Ablation Index, also known as VISITAG SURPOINT $^{\mathrm{TM}}$, is a novel lesion-quality marker that improves outcomes in radiofrequency (RF) catheter ablation of atrial fibrillation $(\mathrm{AF})$. There is no direct evidence on the comparative effectiveness of RF ablation with Ablation Index and cryoballoon (CB). The

Enhanced Digital Features To view enhanced digital features for this article go to https://doi.org/10.6084/ m9.figshare.11205407.

Electronic Supplementary Material The online version of this article (https://doi.org/10.1007/s12325019-01173-4) contains supplementary material, which is available to authorized users.

A. Hussein

St. Louis University, St. Louis, MO, USA

D. Gupta

Liverpool Heart and Chest Hospital, Liverpool, UK

T. De Potter

Cardiovascular Center, OLV Hospital, Aalst,

Belgium

P. Spin

Cornerstone Research Group Inc, Sydney, NS,

Canada

K. Eaton · D. Grima · L. Patel

Cornerstone Research Group Inc, Burlington, ON, Canada objective of the present study was to conduct a matching-adjusted indirect comparison (MAIC) using individual patient-level data (IPD) to compare the effectiveness of RF ablation with Ablation Index to that of $\mathrm{CB}$ on recurrence of atrial arrhythmias 12 months after catheter ablation in patients with paroxysmal AF (PAF).

Methods: Individual patient-level data for RF ablation with Ablation Index were obtained from two studies: Solimene et al. [J Interv Card Electrophysiol 54(1):9-15, 2019] and Hussein et al. [J Cardiovasc Electrophysiol 28(9):1037-1047, 2017]. Comparable CB studies identified from a systematic literature review were pooled. Prognostic variables for adjustment were ranked a priori by several practicing electrophysiologists. In the absence of a common treatment arm between the Ablation Index and CB studies, an

\author{
L. Goldstein $(\bowtie)$ \\ Biosense Webster Inc, Irvine, CA, USA \\ e-mail: lgoldste@ITS.JNJ.com \\ M. Velleca \\ Johnson \& Johnson Medical S.p.A., Pomezia, Italy \\ G. Costa \\ Johnson \& Johnson Medical NV, Diegem, Belgium \\ G. Stabile \\ Clinica Montevergine Mercogliano (AV), Casa di \\ Cura San Michele, Maddaloni, CE, Italy
}


unanchored MAIC was conducted. Primary analysis compared the Solimene et al. study to pooled CB studies. A secondary analysis compared pooled RF ablation with Ablation Index studies to pooled CB studies. Several scenario and sensitivity analyses were conducted.

Results: Primary analyses showed statistically significant reductions in the rate of arrhythmia recurrence with RF ablation with Ablation Index compared to $\mathrm{CB}$ in unmatched, unadjusted (HR 0.50, 95\% CI 0.27-0.95) and matched $(0.42,0.21-0.86)$ analyses. Greater reductions in the rate of arrhythmia recurrence that favored RF ablation with Ablation Index were observed after matching and adjusting for age $(0.41,0.20-0.85)$, age and left ventricular ejection fraction $(0.37,0.16-0.88)$, and age, sex, and left ventricular ejection fraction $(0.30$, 0.13-0.71). Secondary and sensitivity analyses showed similar reductions.

Conclusions: Radiofrequency ablation with Ablation Index was associated with reductions in recurrence of atrial arrhythmias at 12 months compared to $\mathrm{CB}$ in unmatched and unadjusted, matched, and matched and adjusted comparisons.

Keywords: Ablation Index; Atrial fibrillation; Catheter ablation; Contact force; Matchingadjusted indirect comparison; Radiofrequency ablation; VISITAG SURPOINT ${ }^{\mathrm{TM}}$ Module

\section{Key Summary Points}

Atrial fibrillation (AF) ablation is routinely performed with radiofrequency (RF) and cryoballoon (CB) catheters.

There are no studies that directly compare the effectiveness of the latest RF and CB ablation technologies.

We conducted a matching-adjusted indirect comparison (MAIC) using individual patient-level data (IPD) to compare the effectiveness of ablation with $\mathrm{RF}$ and Ablation Index to that of $\mathrm{CB}$ on recurrence of atrial arrhythmias in patients with paroxysmal AF (PAF) at 12-month follow-up.
Results showed that RF ablation with Ablation Index was associated with reductions in recurrence of atrial arrhythmias at 12-month follow-up compared to $\mathrm{CB}$.

This study provides key decision-makers with high-quality, comparative evidence of the most advanced catheter ablation devices for the treatment of AF.

\section{INTRODUCTION}

Pulmonary vein isolation (PVI) using catheter ablation is a treatment option that is recommended by clinical guidelines and is commonly used first line or second line after antiarrhythmic drug (AAD) therapy for patients with symptomatic atrial fibrillation (AF) [1-4]. Several catheter ablation technologies for PVI are available, with evidence demonstrating that both catheter ablation using radiofrequency (RF) ablation or cryoballoon (CB) ablation technology to achieve PVI effectively reduces the recurrence of AF episodes [5-7].

Recently, a novel lesion-quality marker to guide RF ablation, Ablation Index also known as VISITAG SURPOINT ${ }^{\mathrm{TM}}$, was introduced to the market. The use of Ablation Index has been associated with significant reductions in the incidence of acute pulmonary vein reconnection and recurrence of atrial arrhythmias at 12 months when used with THERMOCOOL SMARTTOUCH ${ }^{\mathrm{TM}}$ and THERMOCOOL SMARTTOUCH $^{\mathrm{TM}}$ SF contact force-guided RF ablation compared to contact force-guided RF ablation alone [8-11]. There are no studies that directly compare the effectiveness of THERMOCOOL SMARTTOUCH ${ }^{\mathrm{TM}} /$ THERMOCOOL SMARTTOUCH $^{\mathrm{TM}} \mathrm{SF}$ with Ablation Index (STAI) to that of $\mathrm{CB}$ in AF ablation. Naïve comparison (i.e., unmatched and unadjusted) of studies of catheter ablation with STAI and CB ablation suggest that STAI may provide superior outcomes. Thus, the objective of the present study was to evaluate the impact of STAI on recurrence of atrial arrhythmias 12 months after 
catheter ablation compared to that with CB in patients with paroxysmal AF (PAF) using individual patient-level data (IPD) within a matching-adjusted indirect comparison (MAIC). An MAIC is a method of indirect treatment comparison (ITC) that can be used to adjust for the influence of between-study differences in protocol and trial populations on study outcomes. This article is based on previously conducted studies and does not contain any studies with human participants or animals performed by any of the authors.

\section{METHODS}

\section{Primer on MAICs}

MAICs use IPD to match the patient cohort in one study to that in another, and then to adjust for between-study differences in patient populations. The goal of matching and adjustment is to reduce differences in prognostic factors (i.e., patient characteristics that are associated with AF) and treatment-effect modifiers (i.e., patient characteristics that may affect the effectiveness of catheter ablation on AF) between the analysis populations [12]. Matching refers to the removal of patients from one trial who would not have been included in the comparator trial. Adjusting refers to reweighting patients using inverse propensity score weighting to reduce differences in baseline characteristics (e.g., sex, mean age) [12]. MAICs can be used to conduct either an "anchored" indirect comparison, when there is a common comparator arm between studies (e.g., sham procedure or older technology), or an "unanchored" indirect comparison, in the absence of a common comparator arm between studies [12].

\section{Identification of Studies for MAIC Analyses}

At the initiation of this study, four studies were published on STAI in patients with PAF $[8,9,13,14]$. Individual patient-level data on STAI were available for reanalysis for two of the investigator-initiated studies $[8,9]$. The Hussein et al. [9] trial was a prospectively collected, propensity-score matched analysis comparing STAI to historical THERMOCOOL SMARTTOUCH $^{\mathrm{TM}}$ control that included 43 PAF patients treated with STAI. The Solimene et al. [8] trial was a prospective, single-arm registry that included 132 PAF patients treated with STAI.

A systematic literature review was conducted to identify CB studies in patients with PAF. A search strategy was developed by an experienced medical information specialist in consultation with the review team (Appendix S1). A second information specialist peer-reviewed the search strategy using the PRESS checklist [15]. Searches were conducted on May 23, 2019, in Ovid MEDLINE $^{\circledR}$, including Epub Ahead of Print and In-Process and Other Non-Indexed Citations, Embase, and the Cochrane Central Register of Controlled Trials. The search strategy incorporated controlled vocabulary (e.g., "catheter ablation", "atrial fibrillation", "pulmonary veins/surgery"), keywords (e.g., "cryoballoon", "paroxysmal AF", "PVI"), and relevant trade names pertaining to the $\mathrm{CB}$ ablation catheter (e.g., Arctic Front). Vocabulary and syntax were adjusted across the databases. Animal-only studies, opinion pieces, and conference abstracts were removed, where possible, from the results. All searches were limited to the publication years 2010 to the present, and no attempts were made to contact study authors.

\section{Screening and Selection Criteria}

Study screening was conducted by two independent reviewers (TK and $\mathrm{MH}$ ). Conflicts were resolved by consensus through discussion or a third party (LP). Cryoballoon studies were eligible for inclusion based on PICOS criteria (Appendix S2). Prospective studies were eligible if they had at least 50 adults with drug-refractory, symptomatic, PAF who received first-time ablation and used the Arctic Front Advance ${ }^{\mathrm{TM}}$ $\mathrm{CB}$ ablation catheter. A 50-patient minimum was used to limit the number of studies given the large literature base in this area. The Arctic Front Advance ${ }^{\mathrm{TM}}$ ablation catheter was selected as the most relevant CB technology given that it represented the standard of care for $\mathrm{CB}$ at the 
time. Included studies reported time-to-event data on freedom from atrial arrhythmias or recurrence of atrial arrhythmias for at least 12 months after an ablation procedure, excluding events that occurred during a 3-month blanking period. Non-English-language articles were excluded.

\section{Data Extraction}

Data were extracted by a single reviewer (KE) and verified for accuracy by a second reviewer (LP). Data items extracted (where available) included study design, sample size, AF type (i.e., paroxysmal or persistent), AAD status (i.e., on or off AADs), number of catheter ablation procedures performed (i.e., single or multiple), and patient baseline characteristics (e.g., age, sex, left atrial diameter [LAD], left ventricular ejection fraction [LVEF], and other relevant prognostic factors and treatment-effect modifiers). The outcome of interest was freedom from atrial arrhythmias or recurrence of atrial arrhythmias at least 12 months after an ablation procedure. Atrial arrhythmias were defined as $\mathrm{AF}$, atrial flutter, or atrial tachyarrhythmias, as reported in studies.

\section{Methods for MAIC Analyses}

Primary analysis was planned to compare the STAI IPD from Solimene et al. [8] to the pooled CB cohort data and to compare the STAI IPD from Hussein et al. [9] to the pooled CB cohort data. An MAIC using only the Hussein et al. [9] IPD was not conducted as the small sample size of PAF patients $(n=43)$ was insufficient to allow robust matching to the pooled $\mathrm{CB}$ cohort to reduce residual imbalances with the included $\mathrm{CB}$ trials. Potential prognostic factors and treatment-effect modifiers reported in the STAI and CB studies were ranked a priori and independently by four electrophysiologists. Average rankings were calculated and used to order potential prognostic factors and treatment-effect modifiers for scenario analyses.

Patients from the STAI IPD were reweighted on the basis of a propensity score method-ofmoments estimation that incorporates prognostic factors and treatment-effect modifiers reported in all trials [12]. This process ensures balance between trials in the summary statistics (e.g., means, proportions, and standard deviations) of included prognostic factors and treatment-effect modifiers (Fig. 1). Pooled analyses were limited to the minimum set of prognostic factors and treatment-effect modifiers common to all studies pooled in each MAIC. The principal analysis for each comparison of datasets was the analysis that adjusted for the most factors and had an effective sample size of at least 50 .

To estimate absolute events associated with cryoablation, reconstructed IPD representing the time-to-event outcomes from the CB studies were derived from published aggregate data (i.e., Kaplan-Meier curves) using the Guyot algorithm [16]. Observations from the reconstructed IPD for the CB trials were unweighted. After matching and reweighting of STAI IPD, the CB-reconstructed IPD and STAI IPD were combined to facilitate estimating the comparative effectiveness of STAI versus CB for recurrence of atrial arrhythmias. Comparative effectiveness estimates were obtained by fitting Cox proportional hazards regressions using the reweighted IPD set. Estimates of absolute effects are reported as the cumulative proportion of patients who had an arrhythmia recurrence after 12-month follow-up. Estimates of relative effects are reported as a hazard ratio (HR) and confidence intervals (CIs), where CIs that do not cross unity (i.e., 1) are considered statistically significant.

\section{Scenario and Sensitivity Analyses}

Scenario analyses were carried out to assess the rigor of the primary analysis by incrementally eliminating less important (based on clinical expert rankings) prognostic variables and treatment-effect modifiers from the reweighting phase. For each scenario, MAICs were estimated using a new set of weights for the STAI population. Residual imbalances in excluded prognostic factors and treatment-effect modifiers due to partial reporting of these data at baseline across the trials may bias results. To investigate 

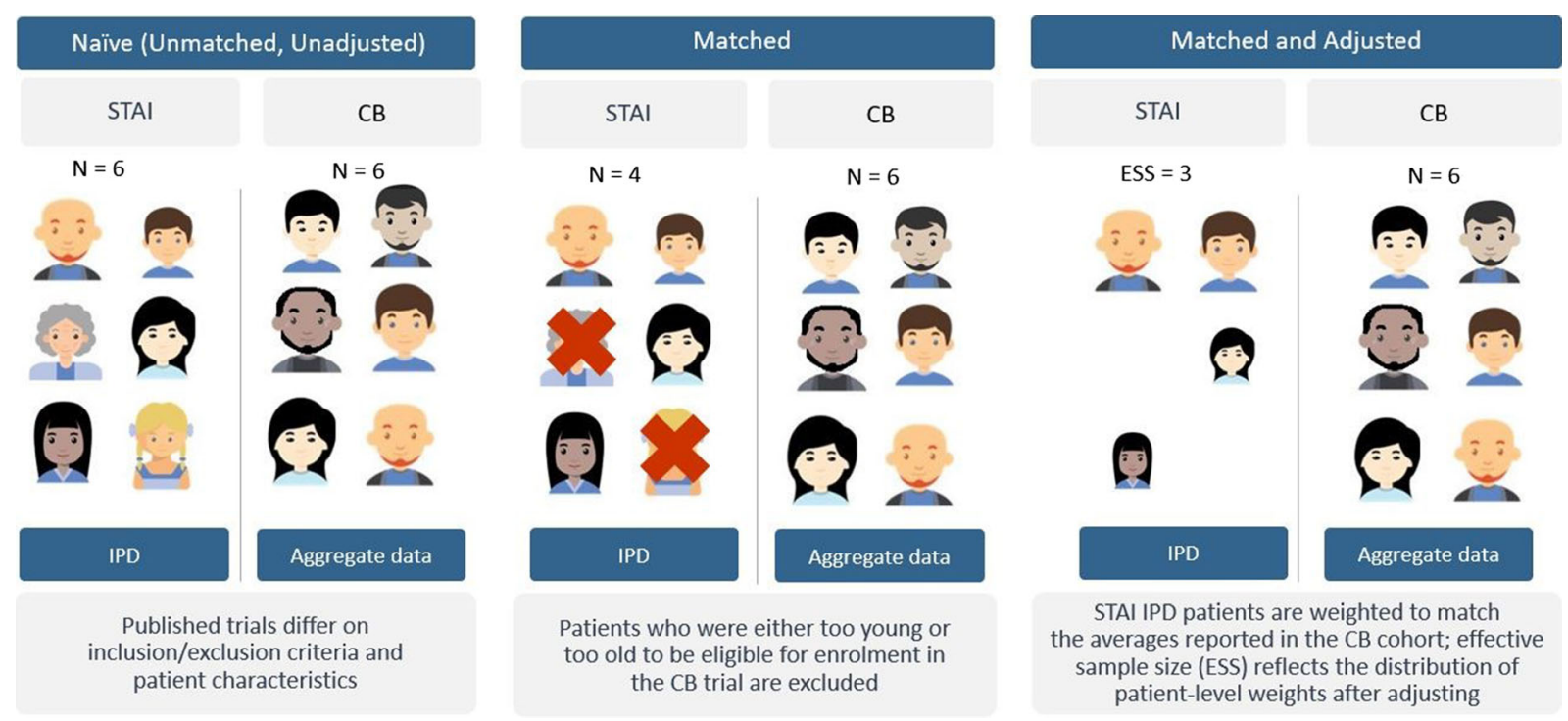

Fig. 1 Matching and adjusting for MAIC. MAIC is a robust method used to reduce differences in patient cohorts from two studies, where IPD from the intervention study is matched and adjusted to aggregate data from a comparator study. Naïve (unmatched, unadjusted): Published trials differ on inclusion/exclusion criteria and patient characteristics. In a naïve comparison, outcomes from the intervention study (i.e., STAI) is compared to those from the comparator study (i.e., $\mathrm{CB}$ ) without considering differences in patient cohorts. Matched: During matching, patients from the STAI IPD that would not have been included in the CB study are removed (e.g., removal of pediatric patients and patients over 90 years old from the STAI study to better match the inclusion criteria

any potential biases, additional factors were incorporated into pairwise MAICs, based on clinician rankings of prognostic factors and treatment-effect modifiers.

\section{RESULTS}

\section{Literature Review Results}

A total of 2186 unique CB records were identified from the literature searches; 68 duplicates were removed (Fig. 2). A total of 1897 citations were excluded during title and abstract review based on PICOS criteria. Of the 221 CB citations identified for full-text review, three were eligible for inclusion [17-19]. The most common of patients 18-90 years old in the CB study). Matched and adjusted: Patients in the STAI IPD are then reweighted to reduce differences in baseline characteristics with the $\mathrm{CB}$ study (e.g., female patients from the STAI study can be down-weighted to simulate the same proportion of female patients in the CB study). This reduces the size of the effective sample (ESS) in the STAI study, which reflects a practical sample size after adjusting. $C B$ cryoballoon, ESS effective sample size, IPD individual patient-level data, $M A I C$ matched-adjusted indirect comparison, $N$ sample size, STAI THERMOCOOL SMARTTOUCH ${ }^{\mathrm{TM}}$ with Ablation Index. Icons altered from Freepik from www. flaticon.com

reasons for exclusion included incomparable study design ( $n=103$; e.g., retrospective studies, case studies, reviews), irrelevant intervention or comparator $(n=54)$, and noncomparable outcomes $(n=42)$ (Fig. 2).

\section{Study Characteristics and Prognostic Factors and Treatment-Effect Modifiers}

Individual CB study cohorts included 50-75 patients with PAF, resulting in a pooled sample size of 175 patients (Table 1 ). The proportion of patients who were male (65-80\%) and the proportion of patients with hypertension (22-36\%) varied across $\mathrm{CB}$ cohorts. Other baseline characteristics were similar among study cohorts (Table 1). Both STAI studies evaluated first-time 


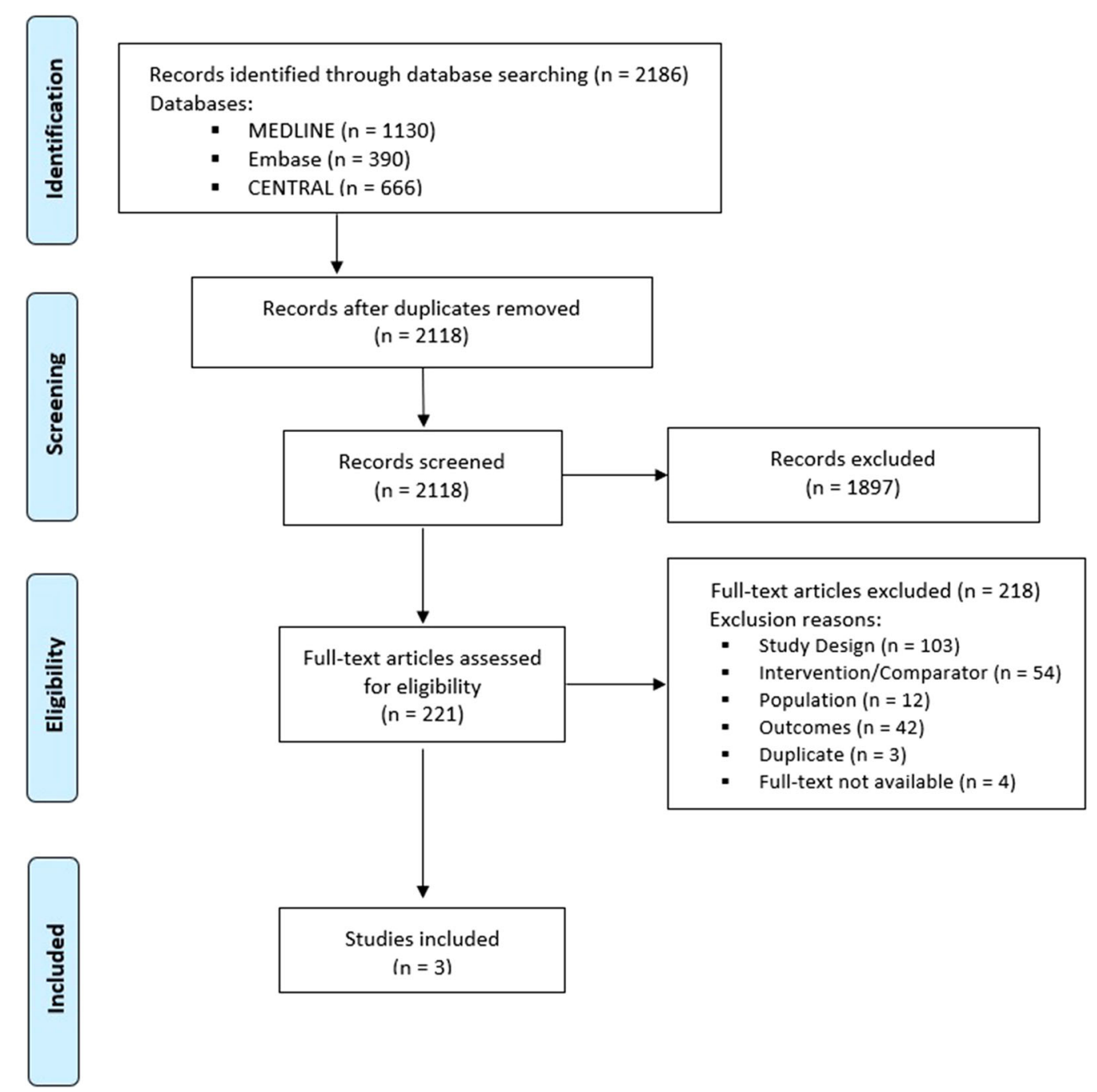

Fig. 2 PRISMA flow diagram for results of the CB literature review. $C B$ second-generation cryoballoon, PRISMA Preferred Reporting Items for Systematic Reviews and Meta-Analyses

ablation outcomes after a single procedure. A total of 175 patients were pooled from STAI trials. Trials differed with regard to the proportion of patients who were male, had PAF, and had diabetes (Table 1).

Before matching and adjusting, several differences in prognostic factors and treatmenteffect modifiers (i.e., patient baseline characteristics) were observed among patients in the pooled CB cohort (referred to as the pooled CB cohort) compared to those in the STAI IPD (Table 1). Standardized mean differences (SMDs) were used to assess imbalances between cohorts, where SMDs greater than 0.1 are considered important. After matching and adjusting, the means and proportions of prognostic factors and treatment-effect modifiers from the STAI IPD were balanced (i.e., SMDs < 0.1 with those in the pooled $\mathrm{CB}$ cohort (Table 2).

\section{MAIC Results}

Results of the primary analysis for STAI IPD from Solimene et al. [8] compared to the pooled CB cohort data and results of the secondary analysis for pooled STAI IPD from Solimene et al. [8] and Hussein et al. [9] compared to the 


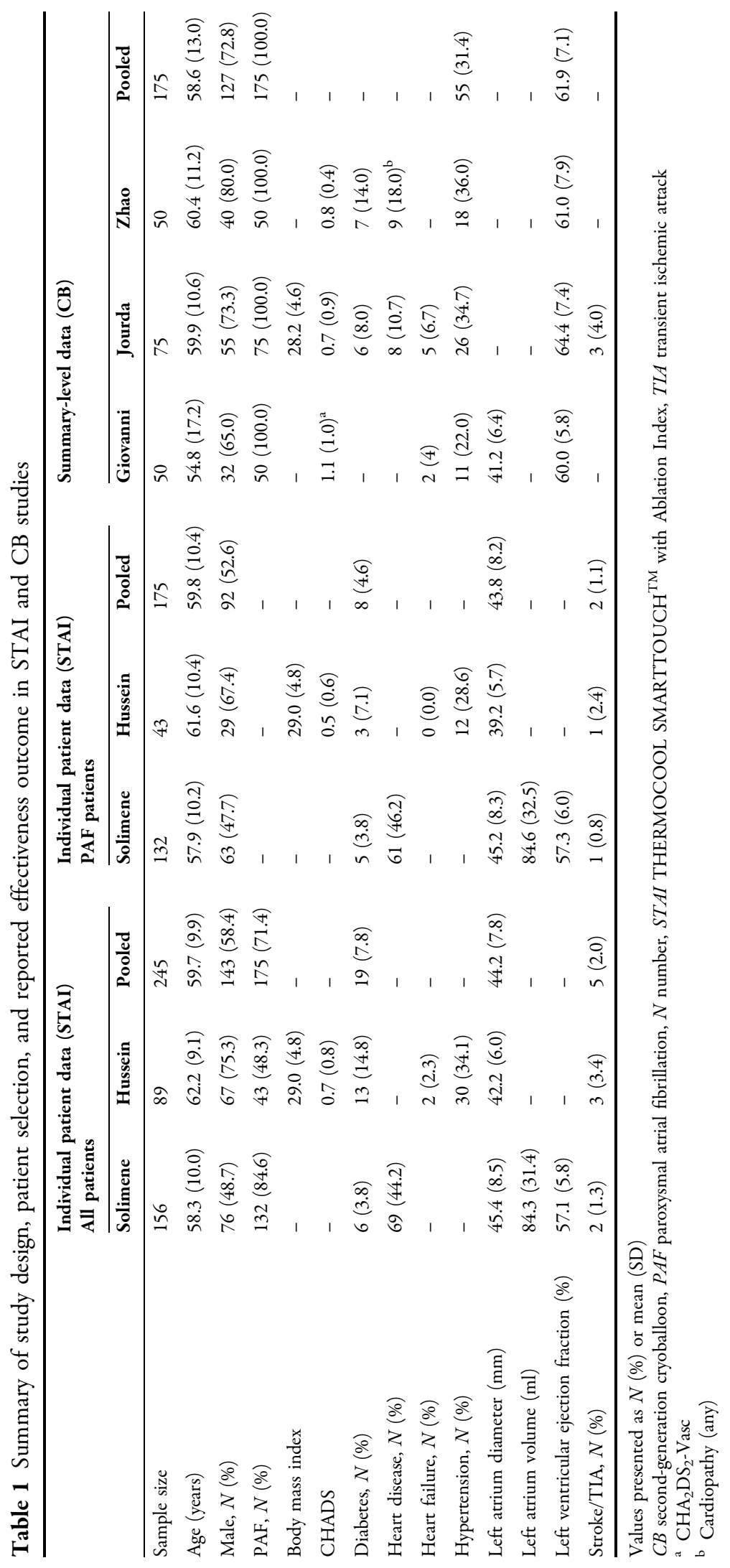


Table 2 Summary statistics for CB trials, pre-matched IPD, post-matched IPD, and matched and adjusted IPD in the primary analysis of recurrence of atrial arrhythmias after a single procedure

\begin{tabular}{lllll}
\hline Variables & Pooled summary-level data $(\mathrm{CB})$ & \multicolumn{3}{l}{ Solimene individual patient data $($ STAI $)$} \\
\cline { 3 - 5 } & & Unmatched & Matched & Matched and adjusted \\
\hline Age (years) & $58.4(11.5)$ & $58.3(10.0)$ & $57.9(10.2)$ & $58.4(11.6)$ \\
$N$ (ESS) & 155 & 156 & 132 & $(125)$ \\
MAIC diagnostics & Proportion of matched IPD set with MAIC weights $=0$ & $0 \%^{\mathrm{a}}$ \\
& Distance of MAIC weights from unity, median $(\mathrm{Q} 1, \mathrm{Q} 3)$ & $-0.69(-0.81,-0.36)$ \\
\hline
\end{tabular}

Values presented as $N(\%)$ or mean $(\mathrm{SD})$

$C B$ second-generation cryoballoon, ESS effective sample size, IPD individual patient data, MAIC matched-adjusted indirect comparison, $N$ number, $Q 1$ first quartile, $Q 3$ third quartile, STAI THERMOCOOL SMARTTOUCH ${ }^{\mathrm{TM}}$ with Ablation Index

a See Appendix S7. MAIC weight histograms

pooled CB cohort data are presented in Figs. 3, 4, and 5. Absolute effectiveness (e.g., cumulative proportion of patients remaining arrhythmia-free after 12 months) is reported in Appendix S3. Results from sensitivity analyses are presented in Appendices S4-S6. Individual comparison of the Hussein et al. [9] IPD to pooled CB data was not conducted because the small sample size of the PAF cohort $(n=43)$ was insufficient to allow robust matching and adjustment to the pooled CB cohort.

\section{Primary Analysis}

Overall, estimates of comparative effectiveness between Solimene et al. [8] STAI and the pooled $\mathrm{CB}$ cohort are significantly in favor of STAI over $\mathrm{CB}$ for arrhythmia recurrence. Naïve comparison of Solimene et al. [8] STAI and pooled CB data showed lower cumulative probabilities of arrhythmia recurrence for STAI $(0.09 ; 95 \%$ CI $0.05-0.14)$ than CB $(0.17 ; 95 \%$ CI $0.12-0.23)$ at 12-month follow-up. This corresponds to a statistically significant $50 \%$ reduction in the rate of arrhythmia recurrence with STAI compared to $\mathrm{CB}$ at 12-month follow-up (HR 0.50; 95\% CI 0.27-0.95). A greater reduction was observed after matching patients, with STAI associated with a significant $58 \%$ reduction in the rate of arrhythmia recurrence compared to $\mathrm{CB}$ at 12-month follow-up (HR $0.42 ; \quad 95 \%$ CI 0.21-0.86). After increasing the equivalence of the two treatment populations by matching patients and adjusting for the common prognostic factor and treatment-effect modifier of age, STAI was associated with a significant 59\% reduction in the rate of arrhythmia recurrence at 12-month follow-up compared to pooled CB (HR 0.41; 95\% CI 0.20-0.85) (Figs. 3 and 4).

Even greater reductions in the rate of arrhythmia recurrence were observed in scenario analyses that adjust for additional common prognostic factors and treatment-effect modifiers, based on a priori clinical ranking (Fig. 3). After adjusting for age and LVEF, STAI was associated with a significant $63 \%$ reduction in the rate of arrhythmia recurrence compared to $\mathrm{CB}$ at 12-month follow-up (HR 0.37; 95\% CI 0.16-0.88). When age, sex, and LVEF were adjusted, a significant $70 \%$ reduction in recurrence rate was associated with STAI when compared to CB (HR 0.30; 95\% CI 0.13-0.71).

\section{Secondary Analysis}

The pooled Solimene et al. [8] and Hussein et al. [9] STAI patient-level data was associated with a lower estimate of the absolute cumulative probability of arrhythmia recurrence $(0.12 ; 95 \%$ CI 0.08-0.16) than the pooled CB cohort $(0.17$; 


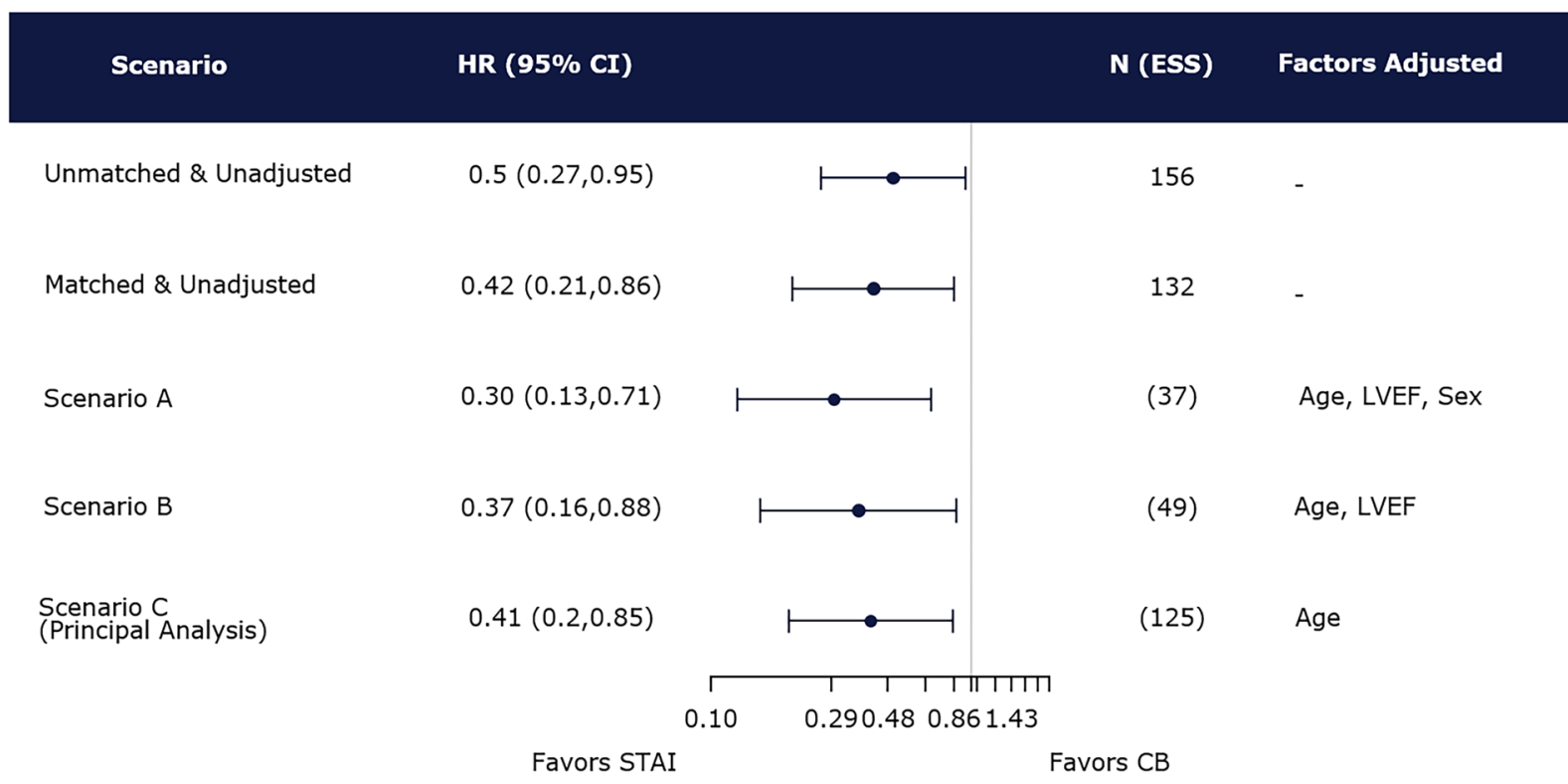

Fig. 3 Hazard ratio for AF recurrence (Solimene IPD vs. pooled $\mathrm{CB}$ cohorts). Forest plot for Solimene IPD vs. pooled $\mathrm{CB}$ cohorts showing the $\mathrm{HR}$ (95\% CI) for arrhythmia recurrence with STAI compared to $\mathrm{CB}$ at 12-month follow-up. Hazard ratio $<1$ represents lower recurrence with STAI than $\mathrm{CB}$, whereas $\mathrm{HR}>1$

95\% CI 0.12-0.23) at 12-month follow-up. As a result, in the naïve comparison, ablation with STAI was associated with a $31 \%$ lower rate of arrhythmia recurrence than $\mathrm{CB}$, but this was not statistically significant (HR 0.69 ; 95\% CI 0.41-1.15). Similar trends were observed after matching patients $(44 \%$ relative reduction in rate, HR 0.56; 95\% CI 0.31-1.01), after matching and adjusting for age and sex (39\% relative reduction in rate, HR 0.61; 95\% CI 0.32-1.15), and in the scenario analysis adjusting for age (43\% relative reduction in rate, HR 0.57 ; 95\% CI 0.31-1.05), which excluded sex on the basis of a priori clinical ranking (Fig. 5).

\section{Sensitivity Analyses Results}

\section{Pairwise Comparison of STAI IPD to Individual CB Studies}

Pairwise comparison of Solimene et al. [8] STAI IPD to individual CB cohorts showed rates of arrhythmia recurrence similar to those in the primary analysis (Appendix S4). represents greater recurrence with STAI than with $C B$. $A F$ atrial fibrillation, $C B$ second-generation cryoballoon, $E S S$ effective sample size, IPD individual patient data, ITC indirect-treatment comparison, $H R$ hazard ratio, STAI THERMOCOOL SMARTTOUCH ${ }^{\mathrm{TM}}$ with Ablation Index

\section{DISCUSSION}

\section{Main Findings}

This study, which used IPD to compare the effectiveness of STAI to that of CB on recurrence of atrial arrhythmias 12 months after catheter ablation in patients with PAF, found that (1) ablation using STAI was associated with reductions in recurrence of atrial arrhythmias compared to CB before and after matching and adjusting for differences between studies and patient characteristics; (2) the results were robust across multiple scenario and sensitivity analyses.

\section{Comparison Between RF and CB}

Pulmonary vein isolation is the cornerstone of all ablation strategies in AF. However, it can be challenging, and there is a significant learning curve in developing the skills needed to safely and effectively perform point-by-point RF 


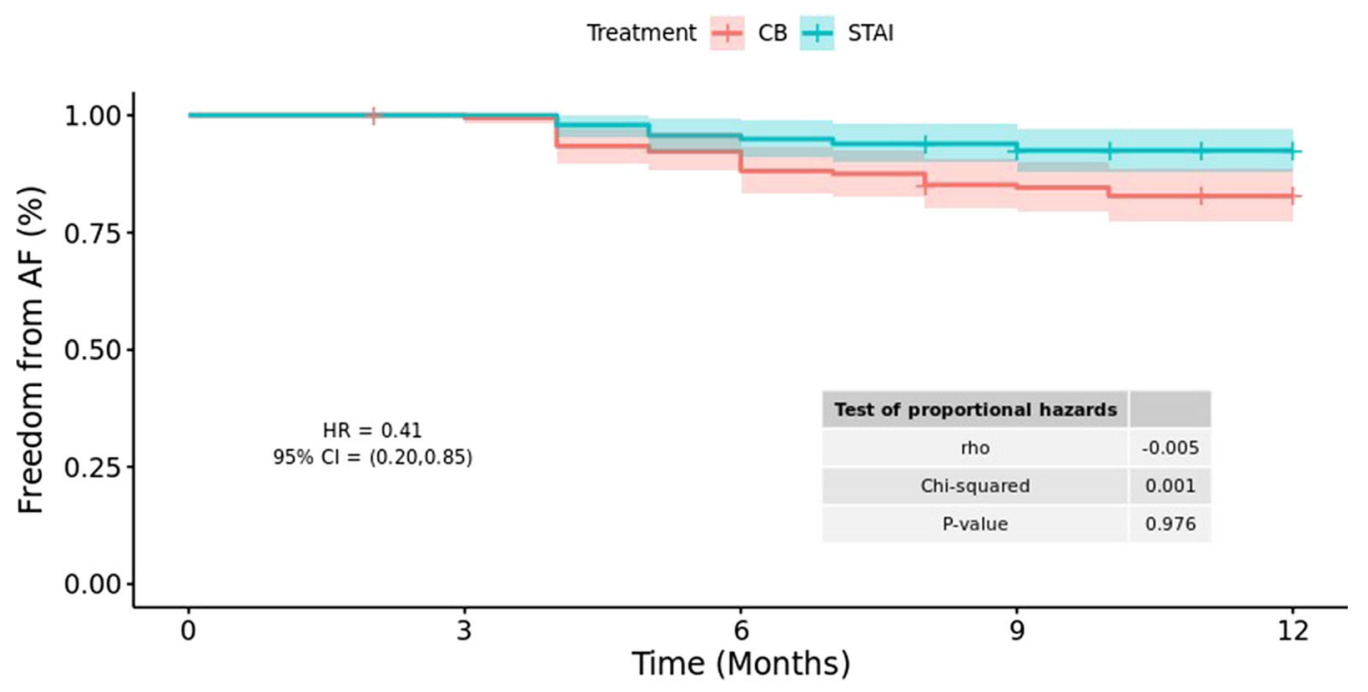

Number at risk

$\begin{array}{ccc}\text { CB } & 175 & 169 \\ \text { STAI } & 128 & 128\end{array}$

Fig. 4 Kaplan-Meier curve of freedom from AF comparing Solimene IPD to pooled CB trials. $A F$ atrial fibrillation, $C B$ second-generation cryoballoon, $C I$ confidence

ablation under 3D electroanatomical guidance. Therefore, novel catheter designs with alternative energy sources have been developed. Many of these novel catheter technologies are balloon-based ablation systems using various energy modalities such as cryoenergy, laser, and RF [2]. Among them, CB ablation is the widest used and recommended in the current guidelines as an alternative to point-by-point RF [1]. Several randomized trials have compared the effectiveness and safety of RF and CB catheters [20-25]; however, of these published studies, few have included advanced catheter ablation technologies [21, 25]. Furthermore, the FIRE AND ICE study, which included some advanced catheter ablation technologies, was not balanced in terms of use of advanced technologies. Specifically, approximately $75 \%$ second-generation CB and only approximately $25 \%$ secondgeneration RF ablation catheters were used; therefore, the study was not sufficiently powered to compare advanced technologies [21, 26].

Recently, use of Ablation Index in RF catheter ablation has shown that it allows for acute and durable PVI followed by a high single-procedure
156

123

interval, $H R$ hazard ratio, KM Kaplan-Meier, STAI THERMOCOOL SMARTTOUCH ${ }^{\mathrm{TM}}$ with Ablation Index

arrhythmia-free survival at 12 months $[8,9,11,13]$. One of the limitations of CB is that it is dependent upon the pulmonary vein (PV) size and anatomy, with the ablated area often being more distal in patients with tubular left common PVs, or in large funnel-shaped veins. Focal RF ablation has the advantage of versatility and is well equipped to deal with variations in $\mathrm{PV}$ anatomy. Prior to the advent of Ablation Index, this advantage of RF was somewhat negated by the high rate of PV reconnection; however, now that Ablation Index-guided has been shown to result in high rates of durable PVI, this superiority has come to the fore.

In the absence of head-to-head trials comparing STAI and CB, we conducted an unanchored MAIC to evaluate the impact of STAI on recurrence of atrial arrhythmias compared to that with CB 12 months after catheter ablation, with corrections for differences in study protocols and populations. The present analysis provides robust, pooled, comparative evidence for the latest generations of catheter ablation devices used in the treatment of PAF, namely the THERMOCOOL SMARTTOUCH ${ }^{\mathrm{TM}}$ Catheter 


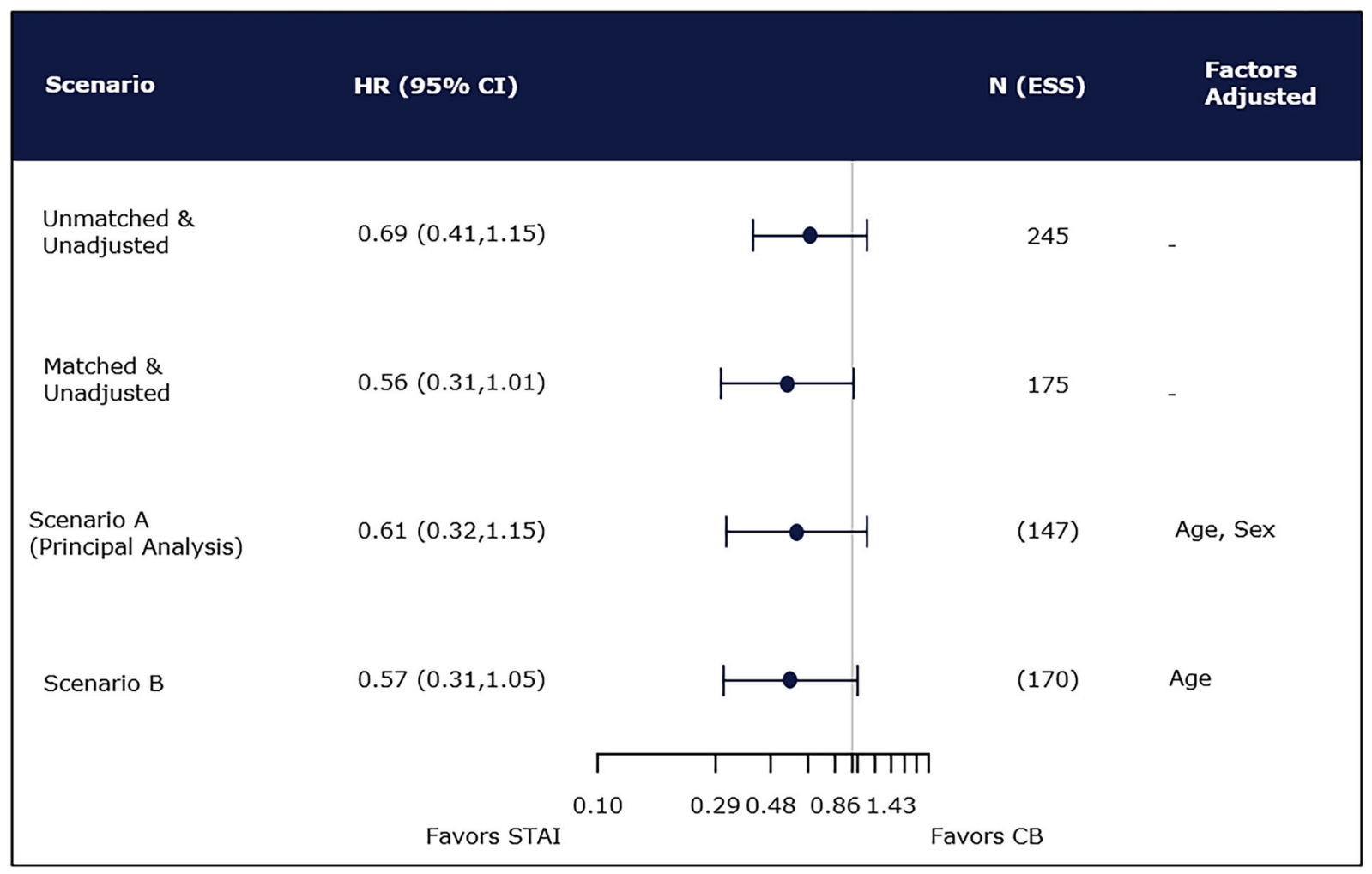

Fig. 5 Hazard ratio for AF recurrence (Solimene + Hussein IPD vs. pooled CB cohorts). Forest plot for Solimene + Hussein IPD vs. pooled CB cohorts showing the HR (95\% CI) for arrhythmia recurrence with STAI compared to $\mathrm{CB}$ at 12 -month follow-up. Hazard ratio $<1$ represents lower recurrence with STAI than $\mathrm{CB}$, whereas

with Ablation Index and the Arctic Front Advance $^{\mathrm{TM}} \mathrm{CB}$ catheter. In the naïve (i.e., unmatched and unadjusted) comparison, better outcomes are apparent with STAI when compared to the pooled CB cohort. Matching and adjustment to minimize differences in study populations between the Solimene et al. [8] IPD and a pooled $\mathrm{CB}$ cohort confirmed and strengthened the outcome difference in favor of STAI (59\% relative reduction in rate; HR 0.41; 95\% CI 0.20-0.85), indicating that betweenstudy differences in patient populations diminished the greater effectiveness of STAI in naïve comparisons. When the Solimene et al. [8] and Hussein et al. [9] IPD were pooled, STAI resulted in a 39\% lower rate of 12-month arrhythmia recurrence than CB (HR 0.61; 95\% CI 0.32-1.15). The reduction in the relative efficacy of STAI when both STAI datasets were
$\mathrm{HR}>1$ represents greater recurrence with STAI than with CB. $A F$ atrial fibrillation, $C B$ second-generation cryoballoon, ESS effective sample size, IPD individual patient data, ITC indirect-treatment comparison, $H R$ hazard ratio, STAI THERMOCOOL SMART$\mathrm{TOUCH}^{\mathrm{TM}}$ with Ablation Index

pooled corresponds with the lower estimate of absolute effectiveness for STAI in this dataset (cumulative probability of arrhythmia recurrence 0.12 ; 95\% CI 0.08-0.16) than in the Solimene et al. [8] IPD set (cumulative probability 0.09 ; 95\% CI 0.05-0.14). This result is not unexpected as the unmatched and unadjusted comparison of STAI IPD for PAF patients from Hussein et al. [9] to the pooled CB cohort data showed no difference in the rate of arrhythmia recurrence between the two treatments (HR 0.98; 95\% CI 0.42-2.29). Although pooled IPD were made more similar to the $\mathrm{CB}$ cohort data through matching on study inclusion/exclusion and adjusting for prognostic factors and treatment-effect modifiers, not all differences between the STAI studies were adjusted for because of lack of reporting or insufficient data to adjust for. For example, the method and 
timing for monitoring arrhythmia recurrence at discharge and throughout the follow-up period varied across studies, potentially influencing reported recurrence rates; however, data that would allow for adjustment of this variable were not available (Appendix S10).

Procedural outcomes were not compared in the present analysis. However, cross-study comparisons suggest that procedure time with Ablation Index-guided ablation is similar to that reported for CB. Reported mean total procedure time ranged from 95 to $175 \mathrm{~min}$ in Ablation Index-guided procedures [10], compared to 90.5-250.5 min in CB procedures [27]. On average, mean fluoroscopy time is shorter with Ablation Index-guided ablation ranging from 5 to $11.9 \mathrm{~min} \mathrm{[10],} \mathrm{in} \mathrm{contrast} \mathrm{to} \mathrm{0-61} \mathrm{min} \mathrm{with}$ CB-guided ablation [27].

\section{Impact of New RF Lesion-Quality Markers}

Contact between catheter tip and tissue, duration, power, impedance, and temperature are all important determinants of lesion size and depth during point-by-point RF catheter ablation [13]. In the past 10 years several new features were introduced to enhance our capability to monitor RF lesion formation, allowing a safe and effective PVI procedure that deploys durable, contiguous, and transmural lesions. Use of contemporary RF catheter ablation technologies has significantly decreased both re-ablation following first PVI and PV reconnection rates at second PVI procedure, as demonstrated in two large, single-center studies $[28,29]$. A recent study confirmed that PV reconnection did not occur in the majority of patients after a single PVI procedure using STAI and the "CLOSE" protocol [30]. These findings may help to explain the results of our analysis which show a significantly lower 12-month recurrence rate after ablation for PAF than has previously been reported [21, 22].

\section{Strengths and Limitations}

A major strength of this study was that prognostic factors and treatment-effect modifiers reported in STAI and CB studies were ranked a priori and independently by electrophysiologists
(Appendix S11), and subsequently adjusted for to reduce any potential bias due to differences in study protocol or populations. However, unanchored MAICs are subject to several inherent limitations. First, the absence of a common comparator within the IPD and comparator trials means that an unanchored MAIC is unable to adjust for differences in prognostic factors and treatment-effect modifiers that cannot be explained by observed differences in prognostic characteristics between trials. Secondly, the ability to reduce imbalances by matching patient populations may be limited by incomplete reporting of eligibility criteria within the comparison trials of interest or by the absence of variables that might otherwise have been used to eliminate patients from the IPD sets who would not have met the eligibility criteria of the comparison study. That is, further adjustment for residual imbalances in patient characteristics is limited to baseline characteristics that are available in the IPD and reported in the comparison trial. In this study, the primary analysis adjusted for one factor (age), which was available in the Solimene et al. [8] IPD and each of the three comparison trials. Adjustment of up to four additional factors (e.g., age, LVEF, diabetes, and sex) common to studies in scenario analyses comparing Solimene et al.'s data [8] to the pooled $\mathrm{CB}$ cohort data and pairwise comparisons of Solimene et al.'s data [8] to data from individual CB studies consistently showed a lower rate of arrhythmia recurrence with STAI than with CB. Lastly, the ability to adjust for prognostic factors and treatment effect modifiers depends on having a sufficient sample size. Although a planned MAIC using only the Hussein et al. [9] IPD was not feasible because of an insufficient sample size of PAF patients, the dataset was pooled with that from Solimene et al. [8], and results showed numerical reductions in the relative rate of recurrence that were similar to the primary analysis.

\section{CONCLUSIONS}

Ablation using THERMOCOOL SMARTTOUCH $^{\mathrm{TM}}$ with Ablation Index was associated with reductions in recurrence of atrial 
arrhythmias at 12-month follow-up compared to $\mathrm{CB}$. Despite limitations inherent to conducting an unanchored MAIC, results were consistent with naïve comparison, and similar across multiple scenario and sensitivity analyses. As such, this analysis provides further evidence available for decision-makers.

\section{ACKNOWLEDGEMENTS}

Funding. This study (including Rapid Service and Open Access Fees) was funded by Biosense Webster, Inc.

Medical Writing and Editorial Assistance. The authors would like to thank Tom Wei, M.BDC of Biosense Webster for general support, Teresa Kangappaden, Hons BMath and Meghan Hughes, PhD of Cornerstone Research Group for systematic literature review support, and Kaitlyn Dawkins, MPH of Cornerstone Research Group for medical writing support. This support was funded by Biosense Webster, Inc.

Authorship. All named authors meet the International Committee of Medical Journal Editors (ICMJE) criteria for authorship for this article, take responsibility for the integrity of the work as a whole, and have given their approval for this version to be published.

Authorship Contributions. LG, MV, and GC contributed to the conception and design of the research and provided critical revisions and intellectual content to the manuscript. AH, DG, TDP, and GS provided substantial intellectual content, and revisions to the manuscript. PS, LP, DG, and KE conducted analyses, discussed the results, drafted the manuscript, and contributed to the final version of the manuscript. All authors provided critical and intellectual feedback of the research, analysis, and manuscript.

Disclosures. Ahmed Hussein is a consultant to Biosense Webster, Inc. Dhiraj Gupta is a consultant to Biosense Webster, Inc. Tom De Potter is a consultant to Biosense Webster, Inc. Giuseppe Stabile is a consultant to Biosense
Webster, Inc. Laura Goldstein is an employee of Biosense Webster, Inc. Maria Velleca is an employee of Biosense Webster, Inc. Graça Costa is an employee of Biosense Webster, Inc. Paul Spin is an employee of Cornerstone Research Group Inc. Kiefer Eaton is an employee of Cornerstone Research Group Inc. Daniel Grima is an employee of Cornerstone Research Group Inc. Leena Patel is an employee of Cornerstone Research Group Inc. Cornerstone Research Group Inc. is a consultant to pharmaceutical and medical device companies, including Biosense Webster, Inc.

Compliance with Ethics Guidelines. This article is based on previously conducted studies and does not contain any studies with human participants or animals performed by any of the authors.

Data Availability. The datasets analyzed during the current study are available from the corresponding author on reasonable request.

Open Access. This article is licensed under a Creative Commons Attribution-NonCommercial 4.0 International License, which permits any non-commercial use, sharing, adaptation, distribution and reproduction in any medium or format, as long as you give appropriate credit to the original author(s) and the source, provide a link to the Creative Commons licence, and indicate if changes were made. The images or other third party material in this article are included in the article's Creative Commons licence, unless indicated otherwise in a credit line to the material. If material is not included in the article's Creative Commons licence and your intended use is not permitted by statutory regulation or exceeds the permitted use, you will need to obtain permission directly from the copyright holder. To view a copy of this licence, visit http:// creativecommons.org/licenses/by-nc/4.0/.

\section{REFERENCES}

1. Kirchhof P, Benussi S, Kotecha D, et al. 2016 ESC guidelines for the management of atrial fibrillation 
developed in collaboration with EACTS. Eur Heart J. 2016;37(38):2893-962. https://doi.org/10.1093/ eurheartj/ehw210.

2. Calkins H, Hindricks G, Cappato R, et al. 2017 HRS/ EHRA/ECAS/APHRS/SOLAECE expert consensus statement on catheter and surgical ablation of atrial fibrillation. Heart Rhythm. 2017;14(10):e275-444. https://doi.org/10.1016/j.hrthm.2017.05.012.

3. January CT, Wann LS, Alpert JS, et al. 2014 AHA/ ACC/HRS guideline for the management of patients with atrial fibrillation: a report of the American College of Cardiology/American Heart Association Task Force on Practice Guidelines and the Heart Rhythm Society. J Am Coll Cardiol. 2014;64(21): e1-76. https://doi.org/10.1016/j.jacc.2014.03.022.

4. January CT, Wann LS, Calkins H, Chen LY, Cigarroa JE, Cleveland JC Jr, et al. AHA/ACC/HRS focused update of the 2014 AHA/ACC/HRS guideline for the management of patients with atrial fibrillation: a report of the American College of Cardiology/ American Heart Association Task Force on Clinical Practice Guidelines and the Heart Rhythm Society. J Am Coll Cardiol. 2019. https://doi.org/10.1016/j. jacc.2019.01.011.

5. Noseworthy PA, Gersh BJ, Kent DM, et al. Atrial fibrillation ablation in practice: assessing CABANA generalizability. Eur Heart J. 2019;40(16):1257-64. https://doi.org/10.1093/eurheartj/ehz085.

6. Packer DL, Mark DB, Robb RA, et al. Effect of catheter ablation vs antiarrhythmic drug therapy on mortality, stroke, bleeding, and cardiac arrest among patients with atrial fibrillation: the CABANA randomized clinical trial. JAMA. 2019;321(13): 1261-74. https://doi.org/10.1001/jama.2019.0693.

7. Marrouche NF, Brachmann J, Andresen D, et al. Catheter ablation for atrial fibrillation with heart failure. N Engl J Med. 2018;378(5):417-27. https:// doi.org/10.1056/NEJMoa1707855.

8. Solimene F, Schillaci V, Shopova G, et al. Safety and efficacy of atrial fibrillation ablation guided by Ablation Index module. J Interv Card Electrophysiol. 2019;54(1):9-15. https://doi.org/10.1007/ s10840-018-0420-5.

9. Hussein A, Das M, Chaturvedi V, et al. Prospective use of Ablation Index targets improves clinical outcomes following ablation for atrial fibrillation. J Cardiovasc Electrophysiol. 2017;28(9):1037-47. https://doi.org/10.1111/jce.13281.

10. Pranata R, Vania R, Huang I. Ablation-index guided versus conventional contact-force guided ablation in pulmonary vein isolation-systematic review and meta-analysis. Indian Pacing Electrophysiol J. 2019. https://doi.org/10.1016/j.ipej.2019.05.001.
11. Hussein A, Das M, Riva S, et al. Use of Ablation Index-guided ablation results in high rates of durable pulmonary vein isolation and freedom from arrhythmia in persistent atrial fibrillation patients. Circ Arrhythm Electrophysiol. 2018;11(9):e006576. https://doi.org/10.1161/CIRCEP.118.006576.

12. Phillippo DA, Ades T, Dias S, Palmer S, Abrams KR, Welton NJ. NICE DSU TSD 18: Methods for population-adjusted indirect comparisons in submissions to NICE, 2016. http://nicedsu.org.uk/wpcontent/uploads/2018/08/Population-adjustmentTSD-FINAL-ref-rerun.pdf. Accessed June 2019.

13. Taghji P, El Haddad M, Phlips T, et al. Evaluation of a strategy aiming to enclose the pulmonary veins with contiguous and optimized radiofrequency lesions in paroxysmal atrial fibrillation: a pilot study. JACC Clin Electrophysiol. 2018;4(1):99-108. https://doi.org/10.1016/j.jacep.2017.06.023.

14. Phlips T, Taghji P, El Haddad M, et al. Improving procedural and one-year outcome after contact force-guided pulmonary vein isolation: the role of interlesion distance, ablation index, and contact force variability in the 'CLOSE'-protocol. Europace. 2018;20(FI_3):f419-27. https://doi.org/10.1093/ europace/eux376.

15. McGowan J, Sampson M, Salzwedel DM, Cogo E, Foerster V, Lefebvre C. PRESS peer review of electronic search strategies: 2015 guideline statement. J Clin Epidemiol. 2016;75:40-6. https://doi.org/10. 1016/j.jclinepi.2016.01.021.

16. Guyot P, Ades A, Ouwens MJ, Welton NJ. Enhanced secondary analysis of survival data: reconstructing the data from published Kaplan-Meier survival curves. BMC Med Res Methodol. 2012;12(1):9. https://doi.org/10.1186/1471-2288-12-9.

17. Jourda F, Providencia R, Marijon E, et al. Contactforce guided radiofrequency vs. second-generation balloon cryotherapy for pulmonary vein isolation in patients with paroxysmal atrial fibrillation-a prospective evaluation. Europace. 2015;17(2): 225-31. https://doi.org/10.1093/europace/euu215.

18. Giovanni GD, Wauters K, Chierchia GB, et al. Oneyear follow-up after single procedure cryoballoon ablation: a comparison between the first and second generation balloon. J Cardiovasc Electrophysiol. 2014;25(8):834-9. https://doi.org/10.1111/jce. 12409.

19. Zhao A, Squara F, Marijon E, Thomas O. Two-year clinical outcome after a single cryoballoon ablation procedure: a comparison of first- and second-generation cryoballoons. Arch Cardiovasc Dis. 2017;110(10):543-9. https://doi.org/10.1016/j. acvd.2017.01.015. 
20. Davtyan K, Shatakhtsyan V, Poghosyan H, et al. Radiofrequency versus cryoballoon ablation of atrial fibrillation: an evaluation using ECG, Holter monitoring, and implantable loop recorders to monitor absolute and clinical effectiveness. Biomed Res Int. 2018;2018:3629384. https://doi.org/10. $1155 / 2018 / 3629384$.

21. Kuck K-H, Brugada J, Fürnkranz A, et al. Cryoballoon or radiofrequency ablation for paroxysmal atrial fibrillation. N Engl J Med. 2016;374(23): 2235-45. https://doi.org/10.1056/NEJMoa1602014.

22. Hunter RJ, Baker V, Finlay MC, et al. Point-by-point radiofrequency ablation versus the cryoballoon or a novel combined approach: a randomized trial comparing three methods of pulmonary vein isolation for paroxysmal atrial fibrillation (the cryo versus RF trial). J Cardiovasc Electrophysiol. 2015;26(12):1307-14. https://doi.org/10.1111/jce. 12846.

23. Luik A, Radzewitz A, Kieser M, et al. Cryoballoon versus open irrigated radiofrequency ablation in patients with paroxysmal atrial fibrillation: the prospective, randomized, controlled, noninferiority FreezeAF Study. Circulation. 2015;132(14):1311-9. https://doi.org/10.1161/CIRCULATIONAHA.115. 016871.

24. Perez-Castellano N, Fernandez-Cavazos R, Moreno J, et al. The COR trial: a randomized study with continuous rhythm monitoring to compare the efficacy of cryoenergy and radiofrequency for pulmonary vein isolation. Heart Rhythm. 2014;11(1): 8-14. https://doi.org/10.1016/j.hrthm.2013.10.014.

25. Gunawardene MA, Hoffmann BA, Schaeffer B, et al. Influence of energy source on early atrial fibrillation recurrences: a comparison of cryoballoon vs. radiofrequency current energy ablation with the endpoint of unexcitability in pulmonary vein isolation. Europace. 2018;20(1):43-9. https://doi.org/ 10.1093/europace/euw307.

26. Kuck KH, Brugada J, Schluter M, et al. The FIRE AND ICE Trial: what we know, what we can still learn, and what we need to address in the future. J Am Heart Assoc. 2018;7(24):e010777. https://doi. org/10.1161/JAHA.118.010777.

27. Patel N, Patel K, Shenoy A, Baker WL, Makaryus AN, El-Sherif N. Cryoballoon ablation for the treatment of atrial fibrillation: a meta-analysis. Curr Cardiol Rev. 2019;15(3):230-8. https://doi.org/10.2174/ $1573403 x 15666181212102419$.

28. Mohanty S, Trivedi C, Gianni C, et al. Procedural findings and ablation outcome in patients with atrial fibrillation referred after two or more failed catheter ablations. J Cardiovasc Electrophysiol. 2017;28(12):1379-86. https://doi.org/10.1111/jce. 13329.

29. Sandorfi G, Rodriguez-Manero M, Saenen J, et al. Less pulmonary vein reconnection at redo procedures following radiofrequency point-by-point antral pulmonary vein isolation with the use of contemporary catheter ablation technologies. JACC Clin Electrophysiol. 2018;4(12):1556-65. https:// doi.org/10.1016/j.jacep.2018.09.020.

30. De Pooter J, Strisciuglio T, El Haddad M, et al. Pulmonary vein reconnection no longer occurs in the majority of patients after a single pulmonary vein isolation procedure. JACC Clin Electrophysiol. 2019;5(3):295-305. https://doi.org/10.1016/j.jacep. 2018.11.020. 